\title{
Reliability and validity of the patellofemoral disability index as a measure of functional performance and subjective pain in subjects with patellofemoral pain syndrome
}

\author{
Mastour Saeed Alshaharani ${ }^{a}$, Everett Bernell Lohman ${ }^{b}$, Khaled Bahjir $^{c}$, Travis Harp ${ }^{d}$, \\ Mansoor Alameri ${ }^{\text {, }}$ Noha S. Daher ${ }^{\mathrm{e}}$ \\ ${ }^{a}$ Rehabilitation Science, School of Allied Health Professions, Loma Linda University, Loma Linda, CA, USA \\ ${ }^{b}$ Department of Physical Therapy, School of Allied Health Professions, Loma Linda University, Loma Linda, CA, USA

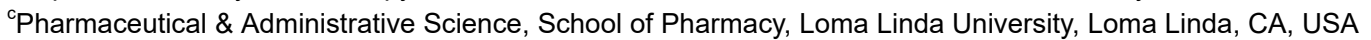 \\ ${ }^{\mathrm{d} A c c e n t C a r e-h o m e ~ H e a l t h c a r e ~ S e r v i c e, ~ D a l l a s, ~ T X, ~ U S A ~}$ \\ ${ }^{\mathrm{e}}$ Allied Health Studies, School of Allied Health Professions, Loma Linda University, Loma Linda, CA, USA
}

Objective: Patellofemoral pain syndrome (PFPS) is a condition that is characterized by patellar discomfort or pain that is aggravated during certain activities such as ascending/descending stairs. The Patellofemoral Disability Index (PDI) was developed to assess the effect of pain on functional activities in individuals with PFPS. The objectives of the current study were to determine the internal consistency, test-retest reliability, and validity of this index.

Design: Cross-sectional study.

Methods: Forty-one subjects who had PFPS with a mean age of $28.8 \pm 5.0$ years and a mean body mass index of $25.6 \pm 4.7 \mathrm{~kg} / \mathrm{m}^{2}$ participated in the study. All subjects were concurrently enrolled in a clinical trial for which they were instructed to complete hamstring-resistance exercises for 4 weeks. Over the course of the intervention, they completed both the PDI and the Oswestry Disability Index (ODI) at baseline after two weeks, and after four weeks. Pearson correlation coefficient was used to assess the criterion validity. Cronbach's $\alpha$ was used to examine the internal consistency. Intraclass correlation coefficients with $95 \%$ confidence interval were computed to examine test-retest reliability.

Results: Subjects' responses within both the PDI and the ODI yielded Pearson correlation coefficient values that were positive and highly significant (range, $0.73-0.97 ; p<0.001$ ). There was a high level of internal consistency (Cronbach's $\alpha \geq 0.8$ ), with the exception of stair climbing (Cronbach's $\alpha=0.65$ ). Intraclass correlation ranged from 0.87 to 0.92 , indicating high levels of test-retest reliability.

Conclusions: The PDI is a valid, reliable, and feasible method of assessing pain and functional ability in patients with PFPS.

Key Words: Anterior knee pain syndrome, Oswestry Disability Index, Patellofemoral Disability Index

\section{Introduction}

Patellofemoral pain syndrome (PFPS) is a condition which is characterized by anterior or retropatellar discomfort or pain that is aggravated during certain activities such as squatting, prolonged sitting, or ascending/descending stairs [1]. PFPS may limit activities of daily living
(ADL) due to a decrease in range of motion, knee stability, and the ability to carry out ordinary activities such as walking, running, sitting down, and squatting. The most recent statistics for PFPS in the United States show a steady increase in cases, with $1.5 \%$ to $7.3 \%$ of all medical care visits being attributed to this condition [2]. Among athletes, approximately $8 \%$ to $33 \%$ of knee-related injuries are due to

Received: 11 April, 2018 Revised: 2 May, 2018 Accepted: 4 May, 2018

Corresponding author: Mastour Saeed Alshaharani (ORCID http://orcid.org/0000-0003-2290-8207)

Rehabilitation Science, School of Allied Health Professions, Loma Linda University, Nichol Hall Room 1605, Loma Linda, CA 92350, USA

Tel: 1-216-527-4585 E-mail: malshaharani@llu.edu

(c) This is an Open-Access article distributed under the terms of the Creative Commons Attribution Non-Commercial License (http://creativecommons.org/licenses/ by-nc/4.0) which permits unrestricted non-commercial use, distribution, and reproduction in any medium, provided the original work is properly cited.

Copyright $@ 2018$ Korean Academy of Physical Therapy Rehabilitation Science 
this condition $[3,4]$.

Self-perceived pain in patients with PFPS is typically assessed using subjective tools such as the Numeric Pain Rating Scale (NPRS), the Anterior Knee Pain Scale, also known as the Kujala Score, and the Oswestry Disability Index (ODI) [5-7]. However, none of these tools are specific to PFPS patients, which shows a need for more appropriate evaluation tools that focus on lower extremity pain and function rather than a general functional assessment.

The ODI is designed to quantify patients' low back pain intensity based on their level of disability [7]. Clinicians use this index in order to get an adequate assessment of patients' disability arising from either lower back pain or knee pain, as the two have been shown to be highly correlated and often occur simultaneously $[8,9]$. The interrelatedness of lower back pain and knee pain has been attributed to several factors such as lumbar lordosis, a cause of lower back pain which may be worsened by degenerative changes in the knee that limit extension of the knee joint [10] and sacral inclination alters the knee flexion angle and is associated with patellofemoral pain [11]. In addition, individuals with lower back pain are simply more prone to experiencing different types of musculoskeletal pain, including knee pain [12].

The Kujala Score was developed in 1993 by Kujala et al. [13] and colleagues with the goal of evaluating the subjective symptoms and limitations in functionality that accompany patellofemoral disorders. There are 8 questions that assess functional limitations and 5 questions that assess impairment, and it has been shown in several studies to be a valid and reliable method of assessing anterior knee pain [14-16]. While the Kujala Score is perhaps the most targeted of these pain measurement tools, it has been reported that the language may be difficult for some patients to understand, especially with regards to the questions concerning knee flexion deficiency, muscle atrophy, and unusual patellar movements [17].

The Patellofemoral Disability Index (PDI) was developed in 1995 and was first published in 1998 by Lohman [18]. This assessment tool provides physical therapists with a method of assessing functional limitations caused by peripatellar pain specifically in patients with PFPS.

The objectives of the current study are to determine the validity of the PDI, measure the internal consistency of the questions regarding sitting, standing, and walking within the PDI, and assess the test-retest reliability of the PDI.

\section{Methods}

\section{Subjects}

This is a cross-sectional study of 41 subjects who reported patellofemoral pain. Subjects were screened by qualified physical therapists under the guidance of a certified orthopedic specialist. The screening exam for patellofemoral pain required subjects to complete the Patellar Apprehension Test [19], Waldron's Test [1], and Clarke's Test [1]. Measurements of patellar gliding/tilting, Q angle, and observations during the step-up and step-down test were also part of the screening exam. Meniscus and ligamentous pathologies were assessed using the McMurray Test [20] and the Anterior Drawer Test [21], respectively.

Recruitment was done through the use of emails, word of mouth, and flyers. In order to be included in the study, subjects must have exhibited patellofemoral pain for at least one month with a pain level of 3 or greater on the NPRS, experienced pain during at least two of the following activities: squatting, ascending/descending stairs, and running, and be between the ages of 18 and 45 years. Subjects who reported any of the following conditions were excluded from the study: traumatic injuries to the knee join/lower limbs, meniscus lesion or ligamentous-related pathology, neurological disorders, past and current medical history of diabetes, osteoarthritis, osteoporosis or rheumatoid arthritis, and/or use of over-the-counter pain medication during the study period. Subjects were not screened for low back pain. All subjects were briefed on the purpose and methods of the study and were required to sign an informed consent form prior to randomization. The study was approved by the Institutional Review Board at Loma Linda University and was registered at http://clinicaltrials.gov (Registration No. NCT03042559).

\section{Instruments}

The Oswestry Low Back Pain Disability Questionnaire, also referred to as the ODI, is a tool employed by physical therapists in both clinical and research settings to assess a patient's functional disability. It is currently considered one of the principal tools for assessing functional ability and quality of life in patients with low back pain [7]. The ODI consists of 10 items that cover a variety of activities which low back pain may limit, such as pain intensity, personal care, lifting, walking, sitting, standing, sexual activity, sleeping, traveling, and socializing. It also allows subjects to rate their pain level during each distinct activity. Under each 
Table 1. Ranges of Patellofemoral Disability Index questionnaire scores and relationship to levels of disability

\begin{tabular}{cll}
\hline Class & Score $\%$ & \multicolumn{1}{c}{ Disability rating } \\
\hline 0 & 0 & No disability \\
1 & $1-20$ & Minimal disability \\
2 & $21-40$ & Minimal/moderate disability \\
3 & $41-60$ & Moderate disability \\
4 & $61-80$ & Severe disability \\
5 & $81-100$ & Bed-bound or symptom magnification \\
\hline
\end{tabular}

section there are six options from which subjects can select the situation that is most relevant to them. These options correspond to a disability rating of zero (no disability) to 5 (bed-bound or symptom magnification) (Table 1). The maximum score is 100 and scores are reported as a percentage. The lower the total score, the smaller the degree of self-perceived disability.

The PDI questionnaire uses a similar format to the ODI. It is specifically designed to assess how a patient's knee pain has affected his/her ability to manage in everyday life, while the ODI is designed to assess the same outcomes with respect to low back pain. Both indices effectively measure functionality and clinical progress of musculoskeletal pain in the low back and lower extremities, conditions which tend to manifest as coexisting morbidities $[8,9]$.

The PDI includes 10 items about physical activities such as running and jumping, as well as other activities including sitting, kneeling, walking, limping, stair-climbing, squatting, standing, and instability. Like the ODI, there are six options within each category and the total score corresponds to a functional ability level ranging from no disability to complete disability. The maximal possible score is 50 and then multiplied by two in order to have a maximum score of 100 to match the disability score using the ODI scale. The results of this questionnaire also correspond to the disability rating scale in Table 1. This questionnaire differs from the ODI in that it targets activities that knee pain may limit, whereas the ODI covers activities that both knee and back pain may inhibit. It has been shown that knee-specific scales are more valid and reliable for subjects with knee pain when compared to more general health assessment instruments [22].

The present study was carried out in conjunction with a clinical trial in which subjects were prescribed hamstring-resistance exercises with either a knee brace or a sport cord. All subjects completed both the ODI and the PDI at baseline, after two weeks, and after four weeks of intervention.
Table 2. Characteristics of participants $(\mathrm{N}=41)$

\begin{tabular}{lc}
\hline \multicolumn{1}{c}{ Characteristic } & Subject \\
\hline Sex & \\
Female & $20(48.8)$ \\
Male & $21(51.2)$ \\
Affected leg & \\
Right & $23(56.1)$ \\
Left & $18(43.9)$ \\
Age $(\mathrm{y})$ & $28.8(5.0)$ \\
Body mass index $\left(\mathrm{kg} / \mathrm{m}^{2}\right)$ & $25.6(4.7)$ \\
Pain duration $(\mathrm{d})$ & $545(30-5,475)$ \\
\hline
\end{tabular}

Values are presented as $\mathrm{n}(\%)$, mean (SD) or mean (min-max).

\section{Data analyses}

Data was analyzed using the statistical package IBM SPSS Statistics ver. 25.0 (IBM Co., Armonk, NY, USA). Pearson correlation coefficient was used to assess the criterion validity of the PDI at all times during sitting, walking, and standing. Cronbach's $\alpha$ was used to examine the internal consistency of PDI items within each type of activity. Intraclass correlation coefficients (ICCs) with $95 \%$ confidence interval (CI) were computed to examine test-retest reliability. The level significance was set at $p \leq 0.05$.

\section{Results}

Subjects' characteristics at baseline are summarized in Table 2.

\section{Validity}

At baseline, subjects' responses to questions regarding sitting, walking, and standing within both the PDI and the ODI yielded Pearson correlation coefficient that were positive, and highly significant. Pearson correlation coefficient ranged from 0.91 to 0.97 at baseline, 0.78 to 0.90 after two weeks, and 0.73 to 0.90 after 4 weeks ( $p<0.001$; Table 3 )

\section{Internal consistency}

Measures of internal consistency were determined using all ten items within the PDI. Each type of activity was found to have an overall high level of consistency with the other items in the questionnaire (Table 4). Cronbach's $\alpha$ values were $\geq 0.8$, indicating high internal consistency. The exception to this was stair climbing, which yielded an overall alpha value of 0.65 . 
Table 3. Pearson correlation coefficients (r) between Patellofemoral Disability Index and Oswestry Disability Index scores

\begin{tabular}{ccc}
\hline Item & $\mathrm{r}$ & $p$-value \\
\hline Baseline & & \\
Sitting & 0.91 & $<0.001$ \\
Walking & 0.91 & $<0.001$ \\
Standing & 0.97 & $<0.001$ \\
Two weeks & & \\
Sitting & 0.90 & $<0.001$ \\
Walking & 0.78 & $<0.001$ \\
Standing & 0.89 & $<0.001$ \\
Four weeks & & \\
Sitting & 0.77 & $<0.001$ \\
Walking & 0.73 & $<0.001$ \\
Standing & 0.90 & $<0.001$ \\
\hline
\end{tabular}

\section{Test-Retest reliability}

Intraclass correlation of the ten PDI items was assessed at baseline, two weeks, and four weeks. The overall values with $95 \%$ CI ranged from $0.87(0.81-0.92)$ to 0.92 (0.89-0.95), indicating high levels of test-retest reliability (Table 5).

\section{Discussion}

\section{Key results \& interpretation}

Based on our findings, the PDI is a valid tool for assessing patellofemoral pain in subjects with PFPS when compared to ODI (the gold standard). Internal consistency was generally satisfactory, in view of the fact that the Cronbach's $\alpha$ scores for each item were generally high with the exception of stair climbing. Test-retest reliability was also satisfactory, though it should be noted that the ICC was slightly greater at two weeks than at four weeks, suggesting a possible decrease in reliability over time.

\section{Generalizability}

To the best of our knowledge, this is the only study that has been done to ascertain the validity and reliability of the PDI in patients with PFPS. Therefore, it is difficult to make a direct comparison of the present study with similar studies. However, the ODI has been widely adapted to suit a variety of languages and cultures, and these modified tools have been tested for validity and reliability. Like the PDI, these tools were derived from the ODI. Therefore, results from these studies may help to give some context to the findings
Table 4. Internal consistency of the ten Patellofemoral Disability Index items within each type of activity

\begin{tabular}{|c|c|}
\hline Item & $\begin{array}{c}\text { Cronbach's } \alpha \\
(95 \% \text { confidence interval })\end{array}$ \\
\hline \multicolumn{2}{|l|}{ Running } \\
\hline All items & $0.87(0.78-0.93)$ \\
\hline Baseline & 0.87 \\
\hline Two weeks & 0.78 \\
\hline Four weeks & 0.79 \\
\hline \multicolumn{2}{|l|}{ Jumping } \\
\hline All item & $0.79(0.65-0.88)$ \\
\hline Baseline & 0.78 \\
\hline Two weeks & 0.65 \\
\hline Four weeks & 0.71 \\
\hline \multicolumn{2}{|l|}{ Kneeling } \\
\hline All item & $0.79(0.65-0.88)$ \\
\hline Baseline & 0.82 \\
\hline Two weeks & 0.61 \\
\hline Four weeks & 0.71 \\
\hline \multicolumn{2}{|l|}{ Instability } \\
\hline All item & $0.90(0.84-0.95)$ \\
\hline Baseline & 0.90 \\
\hline Two weeks & 0.78 \\
\hline Four weeks & 0.90 \\
\hline \multicolumn{2}{|l|}{ Limping } \\
\hline All item & $0.88(0.80-0.93)$ \\
\hline Baseline & 0.90 \\
\hline Two weeks & 0.76 \\
\hline Four weeks & 0.83 \\
\hline \multicolumn{2}{|l|}{ Stair climbing } \\
\hline All item & $0.65(0.42-0.80)$ \\
\hline Baseline & 0.66 \\
\hline Two weeks & 0.36 \\
\hline Four weeks & 0.64 \\
\hline \multicolumn{2}{|l|}{ Squatting } \\
\hline All item & $0.80(0.67-0.89)$ \\
\hline Baseline & 0.89 \\
\hline Two weeks & 0.71 \\
\hline Four weeks & 0.57 \\
\hline \multicolumn{2}{|l|}{ Sitting } \\
\hline All item & $0.90(0.84-0.95)$ \\
\hline Baseline & 0.88 \\
\hline Two weeks & 0.86 \\
\hline Four weeks & 0.84 \\
\hline \multicolumn{2}{|l|}{ Walking } \\
\hline All item & $0.85(0.75-0.92)$ \\
\hline Baseline & 0.85 \\
\hline Two weeks & 0.77 \\
\hline Four weeks & 0.75 \\
\hline \multicolumn{2}{|l|}{ Standing } \\
\hline All item & $0.87(0.78-0.93)$ \\
\hline Baseline & 0.91 \\
\hline Two weeks & 0.75 \\
\hline Four weeks & 0.80 \\
\hline
\end{tabular}


Table 5. Intraclass correlation coefficient of the ten Patellofemoral Disability Index items within each time point

\begin{tabular}{lccc}
\hline \multirow{2}{*}{ Item } & \multicolumn{2}{c}{ Cronbach's $\alpha(95 \%$ confidence interval $)$} \\
\cline { 2 - 4 } & Baseline & Two weeks & Four weeks \\
\hline All ten item & 0.87 & 0.92 & 0.91 \\
& $(0.81-0.92)$ & $(0.89-0.95)$ & $(0.86-0.94)$ \\
Running & 0.87 & 0.91 & 0.92 \\
Jumping & 0.87 & 0.92 & 0.89 \\
Kneeling & 0.87 & 0.92 & 0.89 \\
Instability & 0.86 & 0.91 & 0.90 \\
Limping & 0.85 & 0.91 & 0.90 \\
Stair climbing & 0.86 & 0.93 & 0.90 \\
Squatting & 0.86 & 0.92 & 0.90 \\
Sitting & 0.85 & 0.91 & 0.89 \\
Walking & 0.86 & 0.92 & 0.89 \\
Standing & 0.86 & 0.91 & 0.89 \\
\hline
\end{tabular}

of the present study.

Vigatto et al. [23] developed and tested the reliability and validity of a Brazilian-Portuguese version for detecting clinical changes in subjects with low back pain. Internal consistency was high (Cronbach's $\alpha=0.87$ ), as was test-retest reliability (Cronbach's $\alpha=0.99$ ). Using the Roland-Morris Disability Questionnaire (RMDQ) as the standard for comparison, the test was also found to be valid $(\mathrm{r}=0.81, p<0.01)$. The Russian version of the ODI has also been shown to have high internal consistency (Cronbach's $\alpha=0.82$ ), though test-retest reliability was not as strong $(\mathrm{ICC}=0.70)$ [24]. The Hungarian ODI has been shown to have excellent internal consistency (Cronbach's $\alpha=0.89$ ), test-retest reliability (ICC $=0.93$ ), and validity, as compared to the physical subscale of the World Health Organization Quality of Life, which was also good $(\mathrm{r}=-0.705, p<0.001)$ [25]. The ODI has also been translated and adapted for those who speak Tamil, and this version also showed high internal consistency (Cronbach's $\alpha=0.92$ ), test-retest reliability (ICC $=0.92$ ), and validity when compared to the RMDQ $(\mathrm{r}=0.82, p<0.01)$ [26]. A modified version of the ODI was tested in a Persian population, and tests of internal consistency and test-retest reliability, and convergent validity yielded much lower values than those of the present study (ICC $=0.43-0.80$, Cronbach's $\alpha=0.69, \mathrm{r}=-0.54, p<0.001$ for highest value, respectively) [27]. Studies assessing the same properties in the Korean and Chinese versions have found similar positive results $[28,29]$. The values obtained from the present study are quite similar to those of the aforementioned studies, supporting the finding that the PDI is a valid and reliable tool.

It is important to note that while the PDI and the Kujala
Score are similar in terms of what they are designed to measure, there are several key differences that make the PDI more apt for assessing functionality in the target population. The utility of the PDI lies principally in the targeted nature of the 10 questions within the index. Patients are asked to assess their condition during $10 \mathrm{ADL}$, among these running, jumping, sitting, kneeling, walking, limping, stair-climbing, squatting, standing, and instability. On the other hand, the Kujala Score consists of 8 questions that evaluate functional limitations and 5 questions that evaluate impairment. Patients are asked to assess their condition through items about limping, support, walking, stair-climbing, squatting, running, jumping, sitting, pain, swelling, subluxations, atrophy of thigh, and flexion deficiency. While there is some overlap, some of the questions within the Kujala score are not specific to patellofemoral pain. Furthermore, it has been reported that subjects may have difficulty understanding some of the jargon that is used in this questionnaire [17]. PDI items in each activity appear to be very specific and intelligible, and they target PFPS-related symptoms including pain level, functional tolerance, and performance of daily activities.

The results of this study must be considered in the context of its potential limitations. The time-frame of the study (4 weeks) was relatively short, and it is possible that changes in either the positive or negative direction may have been observed for test-retest reliability had the study period been extended. Furthermore, study participants were generally sedentary individuals, therefore the results may not be generalizable to athletes who, in particular, are prone to developing PFPS.

Based on our findings, the PDI is a sufficiently valid, reliable, and feasible method of assessing subjective pain and functional ability in patients with PFPS.

\section{Conflict of Interest}

The authors declared no potential conflicts of interest with respect to the authorship and/or publication of this article.

\section{References}

1. Nijs J, Van Geel C, Van der auwera C, Van de Velde B. Diagnostic value of five clinical tests in patellofemoral pain syndrome. Man Ther 2006;11:69-77.

2. Glaviano NR, Kew M, Hart JM, Saliba S. Demographic and epidemiological trends in patellofemoral pain. Int J Sports Phys 
Ther 2015;10:281-90.

3. Kannus P, Aho H, Järvinen M, Niittymäki S. Computerized recording of visits to an outpatient sports clinic. Am J Sports Med 1987; 15:79-85.

4. Devereaux MD, Lachmann SM. Patello-femoral arthralgia in athletes attending a Sports Injury Clinic. Br J Sports Med 1984;18:18-21.

5. Singer B, Singer K. Anterior knee pain scale. Aust J Physiother 2009;55:140.

6. Piva SR, Gil AB, Moore CG, Fitzgerald GK. Responsiveness of the activities of daily living scale of the knee outcome survey and numeric pain rating scale in patients with patellofemoral pain. $\mathrm{J}$ Rehabil Med 2009;41:129-35.

7. Fairbank JC, Pynsent PB. The Oswestry Disability Index. Spine (Phila Pa 1976) 2000;25:2940-52; discussion 2952.

8. Rahbar M, Shimia M, Toopchizadeh V, Abed M. Association between knee pain and low back pain. J Pak Med Assoc 2015;65:626-31.

9. Suri P, Morgenroth DC, Kwoh CK, Bean JF, Kalichman L, Hunter DJ. Low back pain and other musculoskeletal pain comorbidities in individuals with symptomatic osteoarthritis of the knee: data from the osteoarthritis initiative. Arthritis Care Res (Hoboken) 2010;62:1715-23.

10. Murata Y, Takahashi K, Yamagata M, Hanaoka E, Moriya H. The knee-spine syndrome. Association between lumbar lordosis and extension of the knee. J Bone Joint Surg Br 2003;85:95-9.

11. Tsuji T, Matsuyama Y, Goto M, Yimin Y, Sato K, Hasegawa Y, et al. Knee-spine syndrome: correlation between sacral inclination and patellofemoral joint pain. J Orthop Sci 2002;7:519-23.

12. Natvig B, Bruusgaard D, Eriksen W. Localized low back pain and low back pain as part of widespread musculoskeletal pain: two different disorders? A cross-sectional population study. J Rehabil Med 2001;33:21-5.

13. Kujala UM, Jaakkola LH, Koskinen SK, Taimela S, Hurme M, Nelimarkka O. Scoring of patellofemoral disorders. Arthroscopy 1993;9:159-63.

14. Ittenbach RF, Huang G, Barber Foss KD, Hewett TE, Myer GD. Reliability and validity of the anterior knee pain scale: applications for use as an epidemiologic screener. PLoS One 2016;11:e0159204.

15. Myer GD, Barber Foss KD, Gupta R, Hewett TE, Ittenbach RF. Analysis of patient-reported anterior knee pain scale: implications for scale development in children and adolescents. Knee Surg Sports Traumatol Arthrosc 2016;24:653-60.

16. Alshehri A, Lohman E, Daher NS, Bahijri K, Alghamdi A, Altorairi N, et al. Cross-cultural adaptation and psychometric properties testing of the arabic anterior knee pain scale. Med Sci Monit 2017;23:1559-82.

17. Watson CJ, Propps M, Ratner J, Zeigler DL, Horton P, Smith SS.
Reliability and responsiveness of the lower extremity functional scale and the anterior knee pain scale in patients with anterior knee pain. J Orthop Sports Phys Ther 2005;35:136-46.

18. Lohman E. Diagnosis and management of patellofemoral pain. In: Godges J, Deyle G, Iglarsh A, Richardson J, editors. Orthopaedic physical therapy clinics of North America: lower quadrant: evidence-based description of clinical practice. Philadelphia: W.B. Saunders Company; 1998. p. 367-96.

19. Ahmad CS, McCarthy M, Gomez JA, Shubin Stein BE. The moving patellar apprehension test for lateral patellar instability. Am J Sports Med 2009;37:791-6.

20. Dervin GF, Stiell IG, Wells GA, Rody K, Grabowski J. Physicians' accuracy and interrator reliability for the diagnosis of unstable meniscal tears in patients having osteoarthritis of the knee. Can J Surg 2001;44:267-74.

21. Kim SJ, Kim HK. Reliability of the anterior drawer test, the pivot shift test, and the Lachman test. Clin Orthop Relat Res 1995;(317):237-42.

22. Paxton EW, Fithian DC, Stone ML, Silva P. The reliability and validity of knee-specific and general health instruments in assessing acute patellar dislocation outcomes. Am J Sports Med 2003;31:487-92.

23. Vigatto R, Alexandre NM, Correa Filho HR. Development of a Brazilian Portuguese version of the Oswestry Disability Index: cross-cultural adaptation, reliability, and validity. Spine (Phila Pa 1976) 2007;32:481-6.

24. Yu EM, Nosova EV, Falkenstein Y, Prasad P, Leasure JM, Kondrashov DG. Validation of a Russian Language Oswestry Disability Index Questionnaire. Global Spine J 2016;6:636-9.

25. Valasek T, Varga PP, Szövérfi Z, Kümin M, Fairbank J, Lazary A. Reliability and validity study on the Hungarian versions of the Oswestry Disability Index and the Quebec Back Pain Disability Scale. Eur Spine J 2013;22:1010-8.

26. Vincent JI, Macdermid JC, Grewal R, Sekar VP, Balachandran D. Translation of Oswestry Disability Index into Tamil with cross cultural adaptation and evaluation of reliability and validity(§). Open Orthop J 2014;8:11-9.

27. Baradaran A, Ebrahimzadeh MH, Birjandinejad A, Kachooei AR. Cross-cultural adaptation, validation, and reliability testing of the modified Oswestry disability questionnaire in Persian population with low back pain. Asian Spine J 2016;10:215-9.

28. Jeon CH, Kim DJ, Kim SK, Kim DJ, Lee HM, Park HJ. Validation in the cross-cultural adaptation of the Korean version of the Oswestry Disability Index. J Korean Med Sci 2006;21:1092-7.

29. Ma C, Wu S, Xiao L, Xue Y. Responsiveness of the Chinese version of the Oswestry disability index in patients with chronic low back pain. Eur Spine J 2011;20:475-81. 